\title{
Vapor phase penetration measurements with both single and double-pass Schlieren for the same injection event
}

\author{
Raul Payri*, F. J. Salvador, Gabriela Bracho, Alberto Viera \\ CMT Motores Termicos, Universitat Politècnica de València, Spain \\ *Corresponding author: rpayri@mot.upv.es
}

\begin{abstract}
Schlieren imaging has been adopted as a standard optical technique for the analysis of diesel sprays under engine like conditions. A single-pass Schlieren arrangement is typically used for the study of single-orifice nozzles, as vessels with multiple optical accesses regularly allow line of sight visualization. Contrarily, for multi-spray nozzles, measurements are commonly performed through a single optical access, in which case a double-pass arrangement is employed. As a consequence, the light beams pass through the test section twice, increasing the optical sensitivity of the Schlieren setup. However, the impact this has on the macroscopic spray characteristics is still unclear. The scope of this study is to analyze the differences in vapor phase penetration for the same injection event, through high-speed imaging, for both single and double-pass Schlieren configurations. Experiments were carried out with a three hole nozzle with a nominal orifice diameter of $90 \mu \mathrm{m}$, named Spray B from the Engine Combustion Network, using commercially available diesel fuel and in non-reactive conditions. The impact of different injection pressures and chamber densities on the spray captured by each setup was assessed. On the results, vapor phase penetration followed the expected trend found in the literature, as it increases with increasing injection pressure and decreasing chamber density. Comparing the optical setups, vapor phase penetration obtained with the double-pass arrangement was marginally higher. The deviation was observed throughout all tested conditions. Although the discrepancy was approximately constant for different injection pressures and chamber temperature, it increased with increasing density. These results highlight the importance of a proper understanding regarding the limitations of optical diagnostics, in particular for results used in calibration of computational models.
\end{abstract}

\section{Keywords}

Diesel injection, Schlieren, vapor phase penetration

\section{Introduction}

In recent years, the implementation of Schlieren imaging in the experimental field has expanded to numerous applications that include military, industrial and scientific research. As Settles [1] stated, it allows seeing optical inhomogeneities in transparent media, like air. In small scale applications, these optical inhomogeneities can relate to density gradients in the medium through which light beams propagate. More specifically, in the field of diesel injection, a Schlieren setup, coupled with high-speed cameras and a proper image processing methodology, has become an essential tool for the study of vaporizing diesel sprays.

Researchers have used Schlieren imaging to analyze the transient vapor phase of the diesel sprays in both nonreactive and reactive conditions. With this technique it is possible to measure simple macroscopic parameters, like spray tip penetration and spreading angle [2,3], to more complex variables, like ignition delay [4-7] or even lift-off length [8]. It is not only fundamental knowledge to understand how boundary conditions affect these variables, but the experimental data is necessary to validate computational tools, such as 1-D models or computational fluid dynamics (CFD).

The two most common Schlieren configurations are single-pass (SP) [2, 5-9] and double-pass (DP) [3, 10-13]. Typically, the first setup is used for axially drilled single-orifice nozzles, where vessels with multiple optical accesses allow line of sight visualization. Contrarily, the double-pass setup is commonly used for multi-hole nozzles, where the sprays tend to be visualized through a single optical access. Consequently, the light crosses the test section twice, and theoretically the Schlieren sensitivity increases by a factor of two [1]. Subsequently, as each region of the spray contour is determined by the amount of refraction the optical configuration can capture, the boundary observed by each system could differ, because is determined by its sensitivity.

The objective of the present study is to compare two different Schlieren imaging setups and their capabilities in measuring vapor phase penetration. Experiments were carried out using a Spray B injector from the Engine Combustion Network (ECN) dataset (http://www.sandia.gov/ecn/). This is a multi-orifice nozzle in which the three holes are not equally spaced. In consequence, one spray is optically isolated from the others, often referred as the spray of interest. Therefore, it can be visualized both as a single and multi-orifice nozzle for the same injection event. Parametric variations of injection pressure, chamber temperature and density were carried out.

This report is divided into four sections. Followed by this introduction, the experimental facility is briefly mentioned, along with a description of the Schlieren principle, optical setups, and image processing methodology. Then, the results regarding background structures and vapor phase penetration are presented, with a short discussion for each case. In the last section, the main conclusions are drawn. 


\section{Materials and methods \\ Testing facility}

A high pressure and temperature vessel, with three optical accesses, was used. The facility can reach nearly quiescent and steady thermodynamic conditions, relevant to the diesel engine. The test chamber presents constant pressure and flow throughout its section, while a group of compressors, high-pressure reservoirs, and heaters provide the necessary conditions for testing purposes. The facility is explained in more detail in the work of other authors $[3,5]$. The fuel delivery system is made up of commercially available components. A Bosch CP3 pump, powered by an electric engine, supplies high-pressure fuel to a common rail with a pressure regulator driven by a PID controller.

The Spray B nozzle (reference 211200) is thoroughly described in the ECN website (http://www.sandia.gov/ ecn/). It consists of a three orifice nozzle, where only the spray of interest is studied, which is located at $180^{\circ}$ relative to the fuel inlet port. The nozzle outlet diameter is $93.2 \mu \mathrm{m}$, with a $k$-factor of 1.5 , and a nominal inclination angle of $17.5^{\circ}$.

\section{Schlieren principle}

Light rays propagate uniformly through homogeneous media, but they are refracted proportional to the refractive index of the medium they enter [1]. Gladstone and Dale [14] found that there is a linear relationship between the refractive index and gas density, presented in Eqn. 1. Where $n$ is the refractive index, $\rho$ is the gas density, and $k_{G D}$ is the Gladstone-Dale coefficient. Additionally, the angular deflection $\varepsilon$ of a ray in the perpendicular plane $x-y$, of a light beam that is traveling in a direction $z$ can be obtained with the expression in Eqn. 2 [1], where $L$ represents the optical path length, and $n_{0}$ the refractive index of the surroundings.

$$
\begin{aligned}
& n-1=\rho \cdot k_{G D} \\
& \varepsilon_{x}=\frac{L}{n_{0}} \frac{\partial n}{\partial x} \quad, \quad \varepsilon_{y}=\frac{L}{n_{0}} \frac{\partial n}{\partial y}
\end{aligned}
$$

From equations 1 and 2, there is a clear relationship between the changes in density and angular deflection of light rays, that can be visualized through Schlieren imaging with a simple optical setup. In Figure 1.a, where a basic single-pass arrangement is presented, beams from a point light source are parallelized by a lens. Any rays deflected by density gradients in the test section (trajectory $i$ in the figure) are blocked by a slit located at the focal distance of the second lens (or cut-off plane). On the contrary, non-deflected beams ( $i i$ in the figure) can pass through the slit and reach the screen. In theory, this produces a black and white image, because the light source is infinitesimal and located exactly at the focal distance of the first lens. Thus each point in the test section is illuminated by a single light ray. In real practice, sources of illumination are finite. Thus the diagram of the setup is more similar to the one presented in Figure 1.b. Here, the Schlieren object in the test section is illuminated by multiple infinitesimal $\partial j$ light sources. In consequence, the image assembled by the second lens is a composition of sub-images, formed by each bundle of rays from each $\partial j$ source [1]. More importantly, this gives the Schlieren setup a continuous dynamic range, in the form of gray-scale intensities (combination of trajectories $i$ and $i i i$ ), compared to the black and white resolution from the first example.

To resemble a double-pass setup, we introduce a mirror where the Lens* is, in Figure 1.b, thus light rays would travel twice through the Schlieren object. In consequence, they are diverted once again by the density gradients, boosting the deflection angle [1]. Additionally, the optical sensitivity can be controlled by the size of the slit. Reducing the aperture is directly related to a decline in minimum deflection angle not blocked by the cutoff [9].

\section{Optical setup for vapor phase visualization}

Following the principles explained in the previous section, both single and double-pass Schlieren imaging were used to study the vapor phase penetration of a diesel spray. Table 1 presents a summary of the optical setup, and Figure

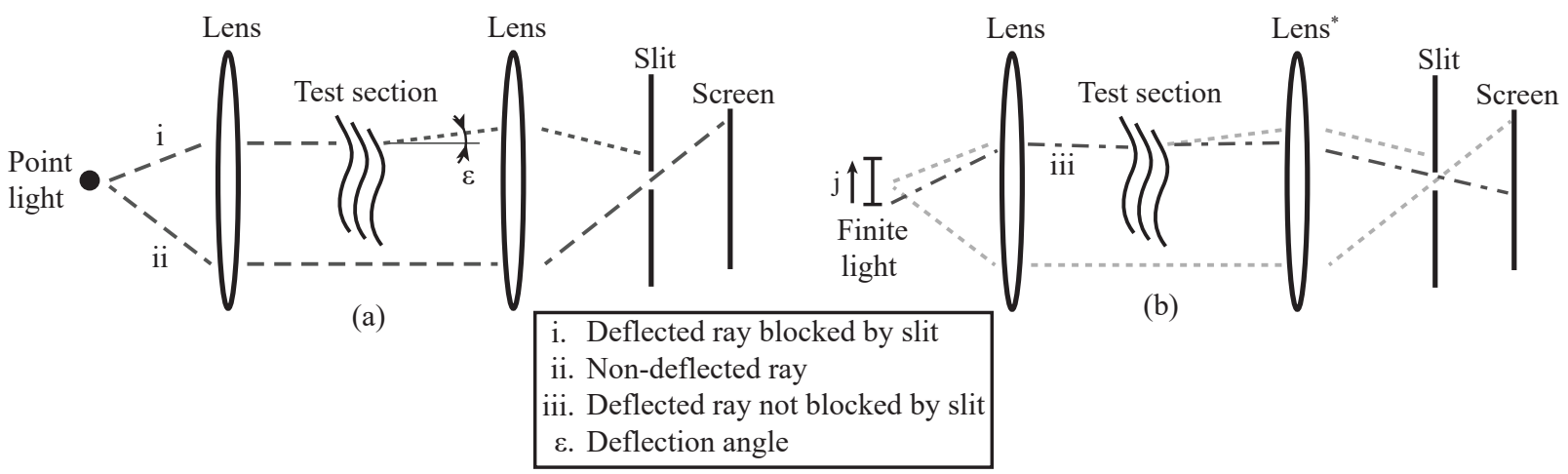

Figure 1. Point (a) and finite (b) light source in Schlieren setup. 
2 the assembly of this configuration for visualization.

For the single-pass arrangement (light trajectory in green), the illumination source was located at the focal distance of a parabolic mirror instead of a regular lens, in contrast to the previous diagram. The angle between the light source and the optical axis of the SP setup was minimized as a result of two factors. Firstly, the long focal distance of the mirror. Secondly, because only one plume is visualized, the source was positioned just above the optical axis, with the minimum angle that allowed the visualization of the spray of interest. As a result, coma aberration was minimized, and the uniformity of the background illumination was improved [1].

In the double-pass configuration (light trajectory in orange), because the spray is visualized through one optical access, a high-temperature mirror is used. Therefore, a beam splitter (50:50) is needed to maintain the optical axis aligned with the other components of the DP setup. As a consequence, only $25 \%$ of the original light reached the cut-off plane. However, the power output of the illumination source was not a constraint, so both setups were calibrated with the same background intensity before measurement.

Additionally, the top left corner of Figure 2 presents the optical perspective inside the test chamber, where the spray of interest is colored in orange. Note that, due to the fact that the high-temperature mirror did not fully enclose the nozzle of the injector, the minimum penetration measurable by the DP setups was $6 \mathrm{~mm}$. Dashed and continuous lines represent the rays before and after passing through the spray, respectively.

The diameter for both diaphragms was set to $4 \mathrm{~mm}$, to have the same sensitivity at the cut-off plane. Previous authors $[3,5,9]$ proved that this diaphragm size provides a proper balance for spray and background segmentation for the Schlieren setups in the facility. Both cameras were set to the same speed, resolution, exposure, and equipped with the same lens.

Table 1. Optical configuration.

\begin{tabular}{ll}
\hline Camera & Photron SA5 \\
\hline Camera lens & Zeiss 100mm \\
\hline Frame rate & $50 \mathrm{kfps}$ \\
\hline Exposure & $10 \mu \mathrm{s}$ \\
\hline Light source & Continous xeon-arc \\
\hline Pixel-mm & $11.4 \mathrm{px} / \mathrm{mm}$ \\
\hline Cut-off & $4 \mathrm{~mm}$ \\
\hline
\end{tabular}
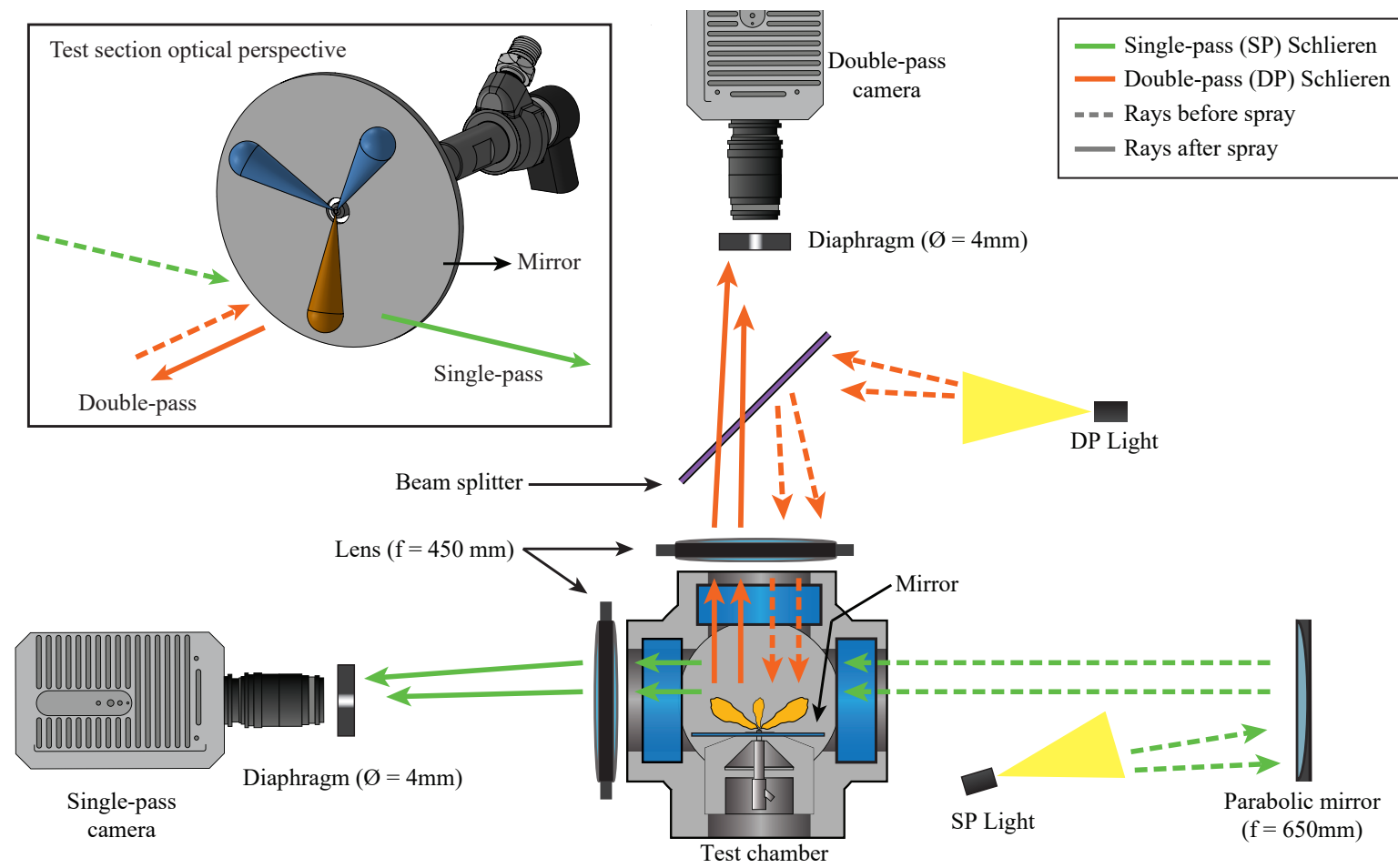

Figure 2. Optical setup used for vapor phase visualization with both single and double-pass Schlieren arrangements. The top right corner presents the optical perspective of the test section, with the spray of interest highlighted in orange. For a better color interpretation of this figure, the reader is referred to the web version of this article. 


\title{
Image processing methodology
}

The procedure used to depict the spray contour is a composition of two extensively used approaches. The core of the segmentation algorithm is based in a fixed threshold intensity-sensitive method $[3,5,10,15]$. But to improve the contour detection capabilities, an image-temporal-derivative approach $[9,16,17]$ was added. Each produces gray-scale images, which are then combined with an adjustable weighted average. This way, the two approaches complement each other. A detailed description of the combined methodology is found in the work of Payri et al. [17]

\section{Definition of the macroscopic spray variables}

With the contour of the spray defined, the next step is to calculate the macroscopic variable for comparison. Regularly, the spray tip penetration is defined as the furthest pixel from the orifice exit, measured in polar coordinates. But, as shown in Figure 2, the spray is observed through different perspectives. The single-pass setup visualizes the real development of the spray, because its axis is parallel to the plane of the camera sensor. Contrarily, the double-pass arrangement sees a projection of the spray evolution, so it is necessary to correct the tip penetration with the inclination angle. Because of the different perspectives, and with the schematics presented in Figure 3 , the following considerations are done:

- The furthest pixel might not be located at the same position for each setup. In consequence, tip penetration (S) could be measured in different geometrical positions of the spray. But the low value of the nozzle inclination angle, and the plume-shaped spray, minimizes the chances of this happening, and its impact on the results.

- The spray tip penetration, measured through the double-pass setup $\left(S_{D P}\right)$, needs to be corrected with the inclination angle $\psi$. But, as mentioned by Payri et al. [18], this angle fluctuates during the injection event.

- The furthest pixel is not contained in the spray axis. Consequently, the correction has to account also the off-axis angle $\delta$.

- Considering the previous points, the variable proposed for comparison is the furthest axial penetration. Since it is parallel to the spray axis, it is always corrected with a constant inclination angle, thus reducing uncertainties due to the spray axis and off-axis angle corrections for the DP spray tip penetration.

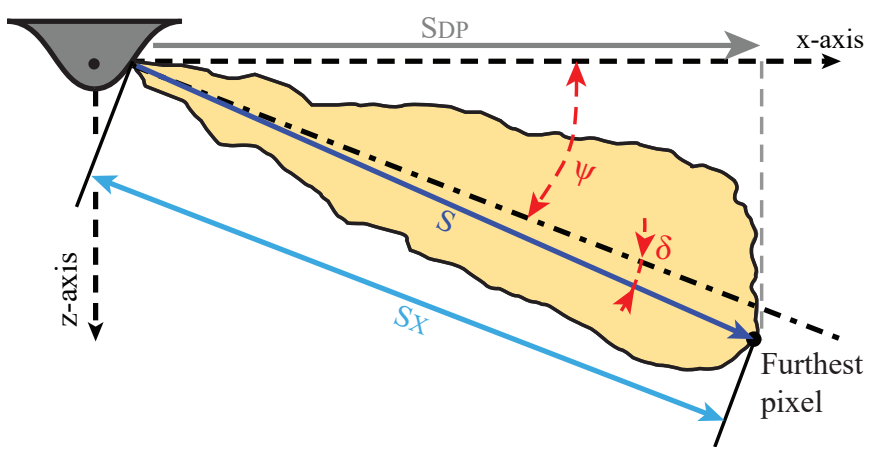

\author{
-.- $\quad$ Spray axis \\ -S Furthest pixel penetration \\ -Sx Furthest pixel axial penetration \\ -SDP Double pass projected penetration \\ $\psi \quad$ Inclination angle \\ $\delta \quad$ Off-axis angle
}

Figure 3. Definition of the spray tip penetration used for comparing single and double-pass Schlieren setups

\section{Data averaging and start of injection}

With the procedures explained in the previous section, the raw results, gathered through high-speed imaging, produce a significant amount of data repetition-wise. Therefore, a moving average technique, described in detail by Payri et al. [19], is used to calculate a single time-dependent curve for the spray tip penetration. For a time instant $t_{i}$, the correspondent penetration value $S\left(t_{i}\right)$ is computed with the linear regression $S=k t+b$. The fitting constants $k$ and $b$ are calculated by solving with $t$ and $S$ consisting of all the data contained in the time interval $t_{i} \pm \Delta t$, with $\Delta t=150 \mu \mathrm{s}$. Subsequently, the average value $\bar{S}$ is estimated by evaluating $t_{i}$ in the regression. This process is repeated for each time-step up to the end of injection. The SOI is calculated by extrapolating the penetration curve to zero, that is $t(S=0)$, with a quadratic fit [10,17-19]. Since the high-temperature mirror in the double-pass setup makes it not possible to observe the first few millimeters of penetration, the SOI was calculated only with the SP data, and then copied to the double-pass data.

\section{Test conditions}

Experimental conditions are listed in Table 2. Values include a parametric sweep in injection pressure, chamber temperature, and density, following ECN recommendations. Experiments were carried out in a non-reactive environment with commercially available diesel fuel. 
Table 2. Test conditions

\begin{tabular}{lll}
\hline Parameter & Value & Units \\
\hline Fuel & Diesel & - \\
\hline Energizing time & 2.5 & $\mathrm{~ms}$ \\
\hline Injection pressure $\left(P_{i n j}\right)$ & $50-100-150$ & $\mathrm{MPa}$ \\
\hline Chamber temperature $(T)$ & $800-900$ & $\mathrm{~K}$ \\
\hline Chamber density $(\rho)$ & $7.6-15.2-22.8$ & $\mathrm{~kg} / \mathrm{m}^{3}$ \\
\hline Gas & Nitrogen & - \\
\hline Repetitions & 10 & - \\
\hline
\end{tabular}

\section{Results and discussion}

\section{Background distribution and spray contrast}

The background of images captured with a Schlieren setup displays a unique structure, because it contains the density gradient information of all the optical path. Not only there is a difference in the distance and trajectory traveled by the light rays inside the test section, but as previously commented, the double-pass arrangement has, theoretically, twice the sensitivity [1]. Figure 4.a-d presents the backgrounds before injection for different chamber temperature and densities. Each column represents images from the SP (left) and DP (right) setups. From this perspective, the hot gas from the heater enters the test section from the right side of the frames.

Focusing in Figure 4.a, the mirror mounted for the DP setup created a stream of flow cooler than the surroundings, marked with a dashed red arrow, and observed throughout the experiments in the SP arrangement. In contrast, this was not visible in the double-pass images, because of the difference in length (thickness) of the optical path that this region represents in each perspective.

The influence of chamber density in the background for each optical setup is presented in Figure 4.a-c. For both systems, increasing the density increases the disturbances captured by the Schlieren, as observed by Pickett et al. [20]. On the contrary, comparing Figures 4.a and 4.d, suggests that chamber temperature produces fewer disturbances in the background, at least for the conditions tested. The marked impact of chamber density in the background is because of its linear scaling with gradients of refractive index [1,20]. On the other side, the effect of temperature depends mainly on its distribution along the test section. Additionally, the double-pass setup presents more complex background structures, with more details in local regions of the frame than the SP configuration. More interestingly, in Figure 4.d, the DP system captures with more detail the hot gases entering the test section. The difference between optical setups on background structures, observed in Figures 4.a-d, are a consequence of at least two factors. On one side, the flow distribution of the hot gases is not strictly equal in each optical plane. On the other, and more importantly, the increased sensitivity for the DP system. Moreover, the optical path length $L$ of the Schlieren object, in this case the test section, is also bigger for the double-pass setup. That is $\approx 250 \mathrm{~mm}$ compared to $\approx 200 \mathrm{~mm}$ for the SP, which is the distance traveled by the light rays inside the test section for each configuration.

The last comparison, Figure 4.e, presents a random time step in the latter part of the injection event for fixed boundary conditions. The clear difference of contrast between spray and background grants more evidence of the higher sensitivity of the double-pass configuration, which provides images with sharper contours and a darker core to the image processing methodology. In consequence, the raw data gathered through the DP setup is less sensitive to differences in thresholding, and the tip region is very well differentiate from the background throughout the injection event, even when local equivalence ratios are low and the density in the region tends to values similar to the surroundings.

\section{Spray tip penetration}

Following the definition from Figure 3, spray tip penetration results, for both Schlieren setups, at different injection pressures are presented in the left part of Figure 5, for fixed conditions of chamber temperature and density. The dashed and continuous line represent both single and double-pass arrangements, respectively. The same line style format is maintained throughout the section. From that figure, both Schlieren setups captured the effect of injection pressure in the tip penetration $[2,17,18]$ equally, as it increases in about the same ratio with increasing rail pressure. More interestingly, the DP configuration depicts higher spray penetration values, being more notably in the later part of the injection event. No effect of the rail pressure in the difference is observed.

The right side of Figure 5, depicts the effect of chamber density on the spray tip penetration. As observed in the previous case, both Schlieren arrangements capture the effect of density in the spray development in agreement with the literature $[10,17]$. However, even though the double-pass setup still depicts higher penetration values, the difference between the optical arrangements is reduced when decreasing the density, and reports almost identical values at the lowest level in the conditions tested.

The effect of the density on the difference between optical setups can be understood by analyzing the sensitivity of each configuration. The deflection caused by the diluted regions of the fuel injected in the test section, given 


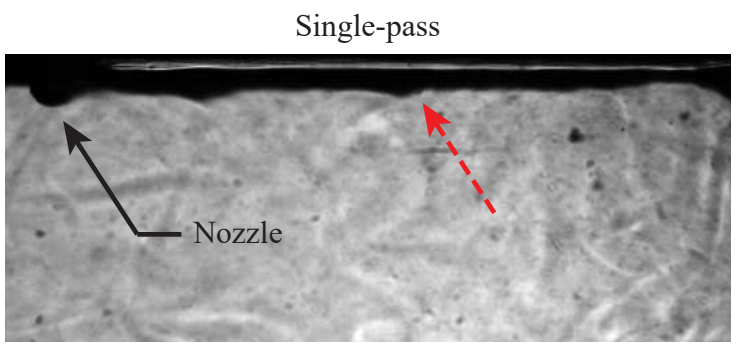

Double-pass

(a) Chamber temperature $=800 \mathrm{~K}$, density $=7.6 \mathrm{~kg} / \mathrm{m}^{3}$
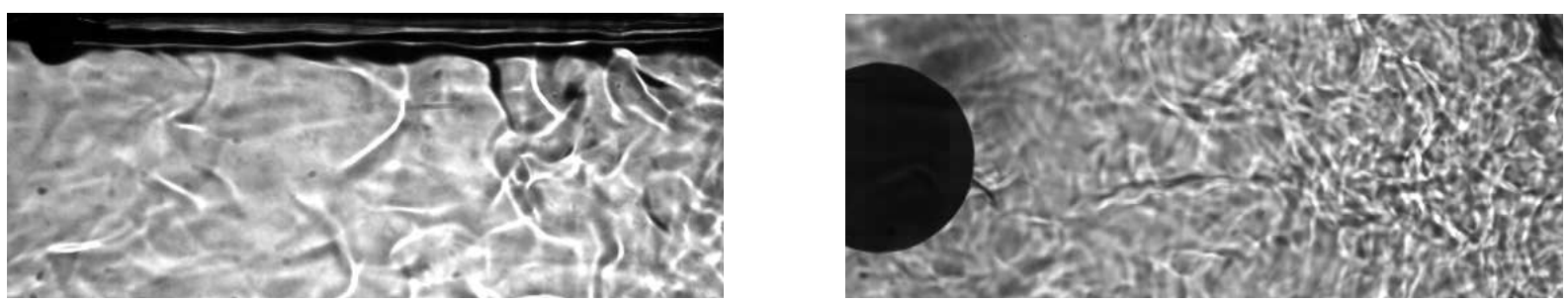

(b) Chamber temperature $=800 \mathrm{~K}$, density $=15.2 \mathrm{~kg} / \mathrm{m}^{3}$.
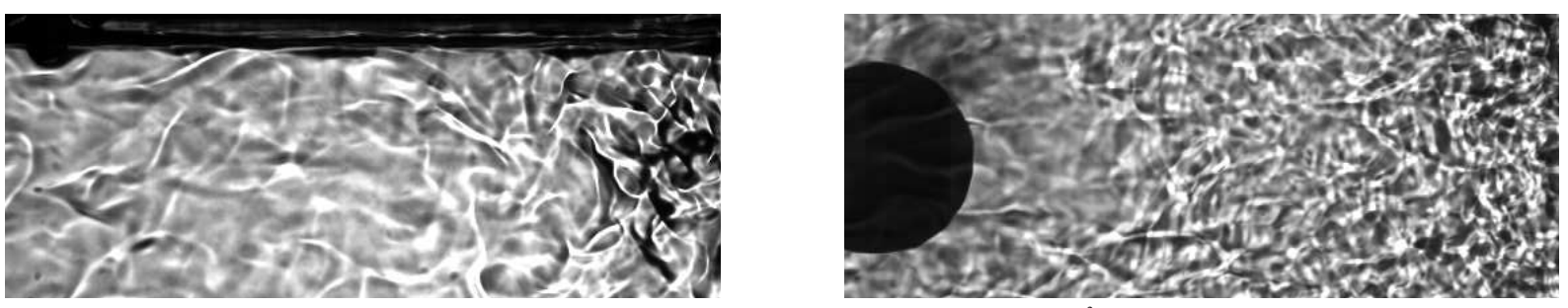

(c) Chamber temperature $=800 \mathrm{~K}$, density $=22.8 \mathrm{~kg} / \mathrm{m}^{3}$.
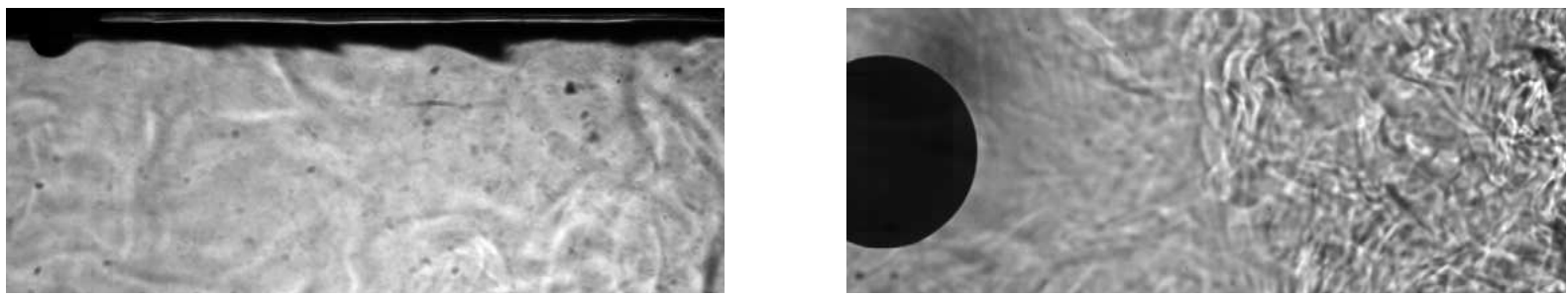

(d) Chamber temperature $=900 \mathrm{~K}$, density $=7.6 \mathrm{~kg} / \mathrm{m}^{3}$.
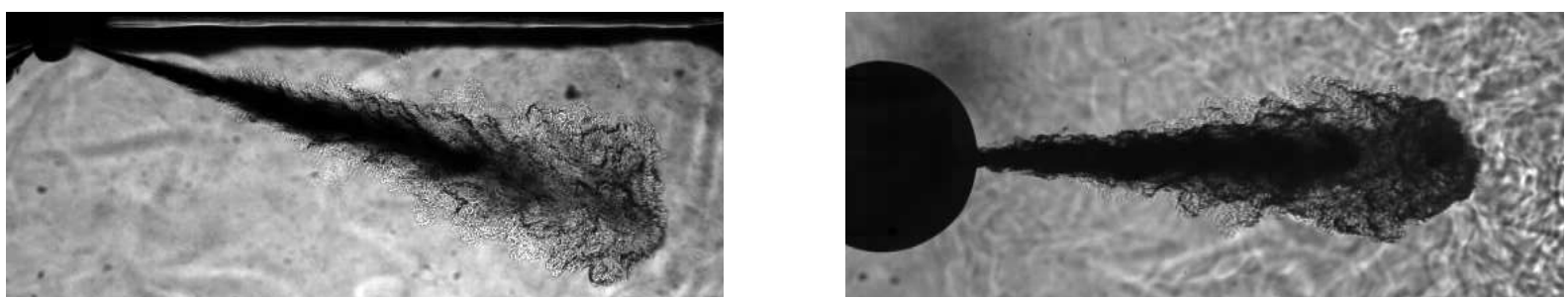

(e) Difference in spray contrast, chamber temperature $=800 \mathrm{~K}$, density $=7.6 \mathrm{~kg} / \mathrm{m}^{3}$.

Figure 4. Differences in background structures and spray contrast for the single (left) and double-pass (right) Schlieren

in Eqn. 2, depends on the refractive index of the surroundings, thus its density (Eqn. 1). Therefore, with the test section at $22.8 \mathrm{~kg} / \mathrm{m}^{3}$, the resultant deflection of a light ray passing through the spray is less than at $7.6 \mathrm{~kg} / \mathrm{m}^{3}$. Furthermore, the density gradients between these diluted regions of the sprays (contours or tip region at a latter part of the injection event) and the surroundings are lower, also producing smaller deflection angle in these areas. Consequently, due to the increased sensitivity of the DP configuration [1], the setup captures "more" spray in the same time step than the SP setup, even though they are visualizing the same injection event.

\section{Further analysis of the two Schlieren configurations}

From the results analyzed in the previous sections, the overall trend found was that the double-pass Schlieren system depicts higher spray tip penetration for all conditions tested. Figure 4.e showed a big difference in contrast and contour sharpness between optical configurations, suggesting that the higher sensitivity in the DP caused this difference in the macroscopic description of the spray. But still, other factors need to be accounted for.

Regarding the optical setup and experimental measurements, both diaphragms were set to $4 \mathrm{~mm}$, resulting in an equal sensitivity at the cut-off plane. Background illumination intensity was calibrated to achieve the same levels before starting the experiments campaign. Additionally, reflections, or double-imaging, were filtered in the cut-off 

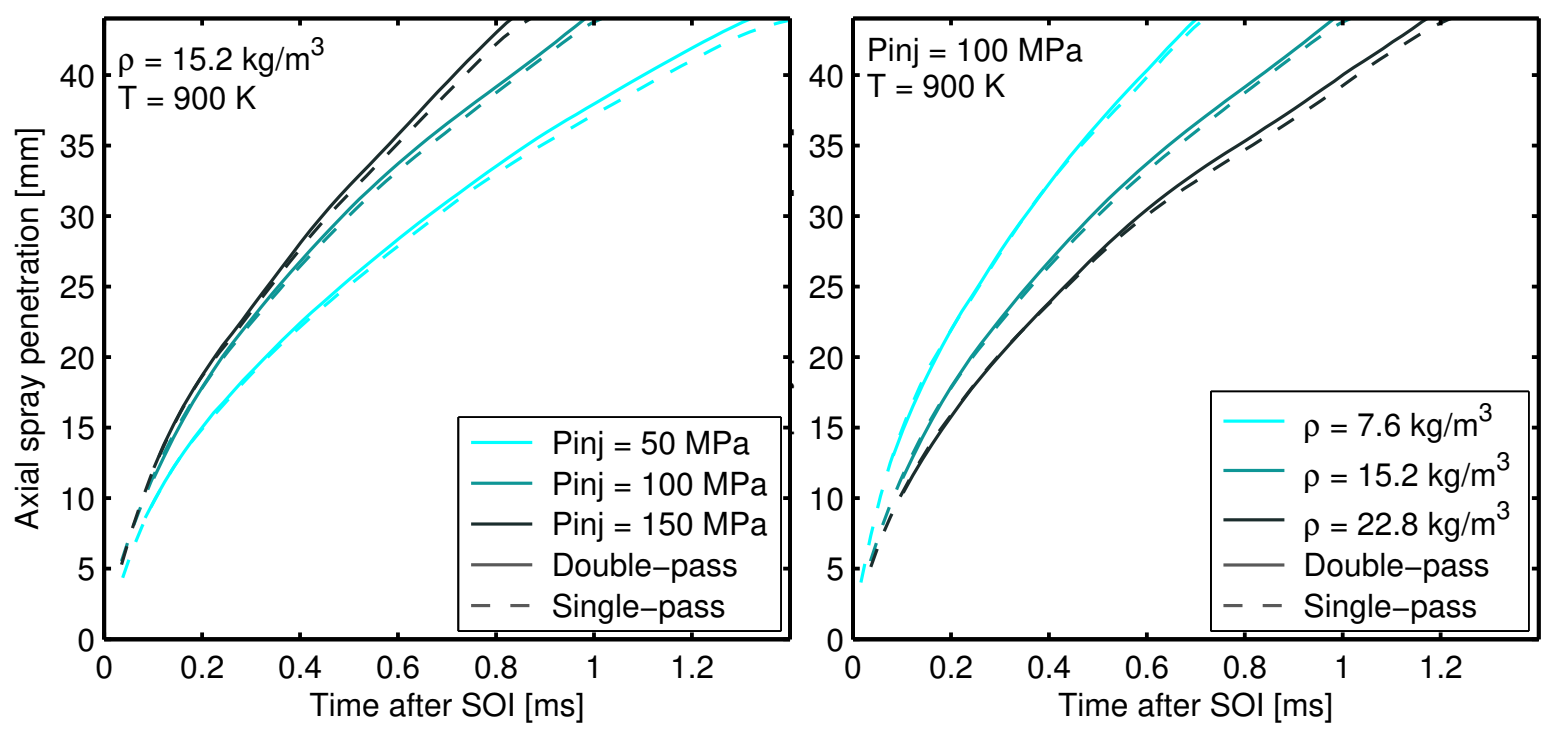

Figure 5. Spray tip penetration for both Schlieren setups at different injection pressures (left) and chamber densities (right).

plane, due to a similar phenomenon as reported by Pastor et al. [11]. Optical accesses typically present small misalignments, caused by differences in the stress of the sealing gaskets, facility assembly, among others, which turn difficult to control. But, curiously, such loss of parallelism becomes an advantage, because these reflections, caused by the optical elements, can be filtered out in the cut-off plane, preventing them from reaching the camera. Regarding the data processing, the same configuration was set for the image processing methodology and moving average technique. But, as stated before, the images provided by the DP system presented more contrast between the spray and the background, that results in easier contour segmentation and less dependence in the thresholding value. In addition, with the spray tip penetration definition used (presented in Figure 3 and previously discussed), the influence of the variable observed for each optical perspective on the final results was diminished.

The differences between setups observed in Figures 4.e and 5, provides some evidence that, because the light rays are passing twice through the test section, boosting its deflection angle, the DP configuration depicts a longer spray. Particularly in regions where the difference between the local density of the spray and its surroundings is low, and the deflection angle is small. Furthermore, the added contrast and sharper contours resulted in images easier to segment with the processing methodology.

\section{Conclusions}

In this research, vapor phase visualization of a diesel spray was performed with both single and double-pass Schlieren imaging setups. Both optical configurations were set up identically in terms of background illumination intensity, camera speed, exposure, pixel to millimeter ratio and diaphragm diameter at the cut-off plane. A three orifice nozzle from the ECN dataset, known as Spray B, was used. Experiments were carried out in a high pressure and temperature vessel, with commercially available diesel, and in a non-reactive environment. A parametric sweep of injection pressure, chamber temperature, and density was performed. The differences between the optical configurations in the background structures and spray development were analyzed for the conditions tested.

The high-temperature mirror used for the double-pass configuration created a stream of flow, cooler than the surroundings, which affected measurements of the single-pass setup near the nozzle (less than 3-4 mm of penetration). On the contrary, this was not visualized by the DP system, due to the differences in the optical path length (thickness) for each perspective. However, because the double-pass mirror did not fully enclose the nozzle, the first 6 $\mathrm{mm}$ of tip penetration were not captured.

The disturbances of background structures, in the frames before injection, notably increase with increasing chamber density, with less influence for increasing chamber temperature, for the range of conditions tested. The marked impact of density on the background distribution was expected because of its linear scaling with gradients of refractive index. The effect of temperature depends on its distribution along the vessel, as the biggest disturbances appear near the inlet of the hot gases to the test section. The double-pass configuration captures more disruptions (gradients) in the background, compared to the single-pass setup.

For all conditions tested, the spray tip penetration results follow the trend expected from other works in the literature. It increases with increasing injection pressure and decreasing chamber density.

Comparing the optical setups, the double-pass system depicts higher spray tip penetration in the latter part of the injection event. No effect from the injection pressure in the difference was observed. On the contrary, decreasing the density reduces the discrepancy between optical configurations, as they present almost identical values at the lowest density tested.

The double-pass setup produces images with a sharper spray contour and more contrast, that are easier to segment from the background. Additionally, due to its higher sensitivity (as bigger deflection angles), the DP system captures 
vaporized fuel in regions where the difference between the local density of the spray and its surroundings is low, and thus the deflection angle is small. Decreasing the chamber density reduces the influence of the sensitivity because the dissimilarities between densities of these local regions are increased.

\section{Acknowledgments}

This research has been partially funded by FEDER and Spanish Ministerio de Economía y Competitividad through project TRA2015-67679-c2-1-R. Additionally, Alberto Viera is supported through the FPI contract 2016-S2-1361 of "Programa de Apoyo para la Investigación y Desarrollo (PAID)" of Universitat Poltècnica de València.

\section{References}

[1] G. S. Settles, Schlieren and Shadowgraph Techniques, Springer Berlin Heidelberg, Berlin, Heidelberg, 2001.

[2] J. D. Naber, D. L. Siebers, Effects of Gas Density and Vaporization on Penetration and Dispersion of Diesel Sprays, SAE Paper 960034 (1996).

[3] R. Payri, F. J. Salvador, J. Gimeno, A. Viera, in: H. Tschöke, R. Marohn (Eds.), 10. Tagung Diesel- und Benzindirekteinspritzung 2016, 1, Springer Vieweg, 2017, pp. 133-152.

[4] R. Payri, J. P. Viera, Y. Pei, S. Som, Experimental and numerical study of lift-off length and ignition delay of a two-component diesel surrogate, Fuel 158 (2015) 957-967.

[5] R. Payri, F. J. Salvador, J. Manin, A. Viera, Diesel ignition delay and lift-off length through different methodologies using a multi-hole injector, Applied Energy 162 (2016) 541-550.

[6] R. Payri, J. P. Viera, V. Gopalakrishnan, P. Szymowitz, The effect of nozzle geometry over ignition delay and flame lift-off of reacting direct-injection sprays for three different fuels, Fuel (2017).

[7] P. M. Lillo, L. M. Pickett, H. Persson, O. Andersson, S. Kook, Diesel Spray Ignition Detection and Spatial/Temporal Correction, SAE Paper 2012-01-1239 (2012) 1-21.

[8] J. V. Pastor, R. Payri, J. M. Garcia-Oliver, F. J. Briceño, Schlieren Methodology for the Analysis of Transient Diesel Flame Evolution, SAE International Journal of Engines 6 (2013) 1661-1676.

[9] J. V. Pastor, R. Payri, J. M. Garcia-Oliver, J.-g. Nerva, Schlieren Measurements of the ECN-Spray A Penetration under Inert and Reacting Conditions, SAE Technical Paper 2012-01-0456 (2012).

[10] R. Payri, J. Gimeno, J. P. Viera, A. H. Plazas, Needle lift profile influence on the vapor phase penetration for a prototype diesel direct acting piezoelectric injector, Fuel 113 (2013) 257-265.

[11] J. V. Pastor, J. M. Garcia-Oliver, J. M. Pastor, L. D. Zapata, Evaporating Diesel Spray Visualization using a Double-pass Shadowgraphy / Schlieren Imaging, SAE technical Paper 2007-24-0026 (2007).

[12] R. Payri, F. J. Salvador, A. Garcia, A. Gil, Combination of visualization techniques for the analysis of evaporating diesel sprays, Energy \& Fuels 26 (2012) 5481-5490.

[13] J. V. Pastor, R. Payri, J. M. Garcia-Oliver, F. J. Briceño, Analysis of Transient Liquid and Vapor Phase Penetration for Diesel Sprays Under Variable Injection Conditions, Atomization and Sprays 21 (2011) 503-520.

[14] J. H. Gladstone, T. P. Dale, Researches on the Refraction, Dispersion, and Sensitiveness of Liquids, Philosophical Transactions of the Royal Society of London 153 (1863) 317-343.

[15] R. Payri, F. J. Salvador, P. Martí-Aldaraví, D. Vaquerizo, ECN Spray G external spray visualization and spray collapse description through penetration and morphology analysis, Applied Thermal Engineering 112 (2017) 304-316.

[16] M. Bardi, R. Payri, L.-M. Malbec, G. Bruneaux, L. M. Pickett, J. Manin, T. Bazyn, C. L. Genzale, Engine Combustion Network: Comparison of Spray Development, Vaporization, and Combustion in Different Combustion Vessels, Atomization and Sprays 22 (2012) 807-842.

[17] R. Payri, J. P. Viera, V. Gopalakrishnan, P. G. Szymkowicz, The effect of nozzle geometry over the evaporative spray formation for three different fuels, Fuel 188 (2017) 645-660.

[18] R. Payri, G. Bracho, P. Marti-Aldaravi, A. Viera, Near field visualization of diesel spray for different nozzle inclination angles in non-vaporizing conditions., Atomization and Sprays 27 (2017) 1-17.

[19] R. Payri, J. Gimeno, M. Bardi, A. H. Plazas, Study liquid length penetration results obtained with a direct acting piezo electric injector, Applied Energy 106 (2013) 152-162.

[20] L. M. Pickett, J. Manin, C. L. Genzale, D. L. Siebers, M. P. B. Musculus, C. A. Idicheria, Relationship Between Diesel Fuel Spray Vapor Penetration/Dispersion and Local Fuel Mixture Fraction, SAE International Journal of Engines 4 (2011) 764-799. 